\title{
Yakov Zeldovich and the Cosmic Web Paradigm
}

\author{
Jaan Einasto \\ Tartu Observatory, \\ Observatooriumi 1, 61602 Tõravere, Estonia \\ email: jaan.einasto@to.ee
}

\begin{abstract}
I discuss the formation of the modern cosmological paradigm. In more detail I describe the early study of dark matter and cosmic web and the role of Yakov Zeldovich in the formation of the present concepts on these subjects.
\end{abstract}

Keywords. cosmology: dark matter, cosmology: large-scale structure of universe, cosmology: theory

\section{Formation of the modern cosmological paradigm}

The modern classical cosmological paradigm was elaborated step by step during the first part of the 20th Century. It was found that there exist stellar systems outside our Milky Way - external galaxies (Öpik (1922), Hubble (1925)). Next it was found that external galaxies are moving away from us, i.e. the Universe is expanding (Hubble (1929)). On the basis of Einstein relativity theory Friedmann (1922) explained the expansion as a property of the infinite universe. The speed of the expansion can be expressed in terms of the Hubble constant, $H_{0}$. Sandage \& Tammann (1975) found a value about $H_{0}=$ $50 \mathrm{~km} \mathrm{~s}^{-1} \mathrm{Mpc}^{-1}$, whereas de Vaucouleurs (1978) and van den Bergh (1972) preferred a value around $H_{0}=100 \mathrm{~km} \mathrm{~s}^{-1} \mathrm{Mpc}^{-1}$. Due to this uncertainty the Hubble constant is often expressed in dimensionless units $h$, defined as: $H_{0}=100 h \mathrm{~km} \mathrm{~s}^{-1} \mathrm{Mpc}^{-1}$.

Another basis of the classical cosmological paradigm is the distribution of galaxies and clusters of galaxies. A photographic survey was made using the 48-inch Palomar Schmidt telescope. Abell (1958) used the Palomar survey to compile a catalogue of rich clusters of galaxies for the Northern sky; later the catalogue was continued to the Southern sky (Abell et al. (1989)). Zwicky et al. (1968) used this survey to compile for the Northern hemisphere a catalogue of galaxies and clusters of galaxies. The galaxy catalogue is complete up to 15.5 photographic magnitude. Both authors noticed that galaxies and clusters of galaxies show a tendency of clustering.

A deeper complete photographic survey of galaxies was made in the Lick Observatory by Shane \& Wirtanen(1967). The Lick counts as well as galaxy and cluster catalogues by Zwicky and Abell were analysed by Jim Peebles and collaborators to exclude count limit irregularities (Soneira \& Peebles (1978)). These data show the apparent (2-dimensional) distribution of galaxies and clusters on the sky. The basic conclusion from these studies is that galaxies are hierarchically clustered. There exist clusters and superclusters of galaxies, but most galaxies form a more-or-less randomly distributed population of field galaxies.

The mean density due to galaxies was determined using the mean luminosity density, and the mean mass-to-luminosity ratio $(M / L)$ of galaxies. Estimates available in the 1950 's indicated a low-density Universe, $\Omega \approx 0.05$. 
This complex of data formed the classical cosmological paradigm. However, the theoretical explanation of the data by the Friedman model was mathematical, it did not consider physical processes in the early universe. Thus in the early 1960's in several centres theorists started to think on the physics of the early universe. Most important developments in this direction were made in Princeton by Jim Peebles, and in Moscow by Yakov Zeldovich and their collaborators.

One of the first step in the study of physical processes was the elaboration of the hierarchical clustering scenario of galaxies by Peebles \& Yu (1970), Peebles (1971). On the other hand, the Moscow team developed the pancake model of structure formation by Zeldovich (1970), and the theory of the Sunyaev-Zeldovich effect in the Cosmic Microwave Background radiation by Sunyaev \& Zeldovich (1969).

To discuss new problems of cosmology and astrophysics Zeldovich organised summer and winter schools. The first of such schools was in the new observatory in Toravere, 1962; later schools were hold in Caucasus winter resorts. Our Tartu cosmology team was invited to Caucasus winter schools in 1972, 1974 and later. Discussions on winter schools started our collaboration with the Zeldovich team.

Also important observational discoveries were made. Penzias \& Wilson (1965) detected the Cosmic Microwave Background radiation. Satellite observatories allowed to detect Xrays from clusters of galaxies, and to find the mass of the hot gas in clusters, as well as the total mass of clusters (Forman et al. (1972)).

These observational and theoretical developments were the basis of the formation of the modern cosmological paradigm. In the following I shall discuss in more detail some aspects of the new cosmological paradigm, related the discovery of the cosmic web. Quite unexpectedly we found that the structure of the cosmic web is closely related to another problem - the nature of the dark matter and its role in the formation of the cosmic web.

\section{Dark matter}

In the middle of 1960's the general attitude of the astronomical community was that the classical cosmological paradigm is in agreement with all observational and theoretical data available. Actually there were some unexplained facts. One of these curious data was the Coma cluster mass paradox. The mass calculated from random motions of galaxies in the cluster was much higher than the expected mass found by adding masses of individual galaxies, as suggested by Zwicky (1933).

Another curious fact was the form of rotation curves of galaxies. As found by Oort (1940), Roberts (1966) and Rubin \& Ford (1970), the rotation curves of spiral galaxies are flat on large galactocentric distances. Since the surface brightness of galaxies falls rapidly on the periphery, flat rotation curves mean, that the mass-to-luminosity ratio rapidly increases on large galactocentric distances. Oort (1940) and Roberts (1966) explained this observation with the assumption that on large distances low-mass stars dominate in galaxies. For some unclear reason these observations were ignored by the astronomical community.

Tartu astronomers have studied methods of modelling the structure of galaxies for years. The first dynamical model of the Andromeda galaxy was calculated by Öpik (1922). Kuzmin(1952), Kuzmin (1956) developed more accurate method of modelling galaxies, and applied the method to our Galaxy.

I helped Kuzmin in calculations and was interested to continue the modelling of galaxies, using more observational data on galactic populations. First I studied carefully methods used by previous authors to calculate mass distribution models of galaxies. To my surprise I found that in most models simple conditions of physical reasonability are not 
satisfied. Most important conditions are: the spatial density must be non-negative and finite, some moments of the density must be finite, in particular moments which define the mass and the effective radius of the model. Thus I found that the only mass distribution profile which satisfies all physical conditions is a generalised exponential model: $\varrho(a)=\varrho_{0} \exp \left(-\left(a / a_{c}\right)^{1 / N}\right)$, where $\varrho_{0}$ is the central density, $a$ is the semi-major axis of the equidensity ellipsoid, $a_{c}$ is the core radius, and $N$ is the structural parameter, which allows one to vary the shape of the density profile. I used this density profile in my model of the Galaxy (Einasto (1965)), and in models of other galaxies. Presently this profile is known as the "Einasto profile".

The central question in modelling galaxies is the calibration of mass-to-luminosity ratios of populations. This can be done using additional independent data. Most important data are velocity dispersions of open and globular clusters with similar photometric properties, assuming that galactic populations have been formed by dissolution of clusters and star associations. To bring data on populations of different age and composition to a coherent system I developed models of evolution of populations, similar to models by Tinsley (1968). To my surprise I discovered that it is impossible to represent rotation curves of galaxies by the sum of gravitational attraction of known stellar populations. The only way to bring kinematical and photometrical data into agreement was to suppose the presence of a new population - corona - with large radius, mass and $M / L$ ratio.

I calculated models with massive coronas for all major galaxies of the Local Group and the Virgo cluster central galaxy, M87. Results were discussed at the First European Astronomy Meeting in Athens, September 1972 (Einasto (1974)). However, observed rotation curves were not long enough to find the mass and the radius of coronas. Thus I continued to think how to find total masses and radii of coronas. Finally I decided to use companion galaxies as mass tracers of giant galaxies. I collected data needed and found that the mass (and the effective radius) of coronas is about ten times larger than the sum of masses of all known stellar populations. The total cosmological density of matter in galaxies including massive coronas is 0.2 of critical cosmological density (Einasto et al. (1974)). A similar density estimate was confirmed by Ostriker et al. (1974).

I reported these results in the Arkhõz Winter School in January 1974. My principal conclusion was, that all giant galaxies have massive coronas, and that coronas cannot have stellar nature. Thus the coronal or dark matter is the principal constituent of the universe, and its nature is not clear. After my talk Zeldovich invited me to his room and asked two questions: Can we find data which give some hints to the physical nature of coronas? Can we find observational evidence which can be used to discriminate between various theories of the formation of galaxies?

To discuss the existence and the physical nature of dark matter, we organised in January 1975 a conference in Tallinn, Estonia (Doroshkevich et al. (1975)). The rumour on dark matter had spread around the astronomical and physics community and all leading Soviet astronomers and physicists attended. Two basic models were suggested for coronae: faint stars or hot gas. It was found that both models have serious difficulties. Neutrinos were also discussed but excluded since they can form only clumps of rich cluster mass, but coronas of galaxies have thousand time lower masses.

The dark matter problem was discussed also in the Third European Astronomical Meeting in Tbilisi in June 1975. In the dark matter session the principal discussion was between the supporters of the classical paradigm with conventional mass estimates of galaxies, and supportes of the new paradigm with dark matter. The most serious arguments in favour of the classical cosmological paradigm were presented by Materne \& Tammann (1976): Big Bang nucleosynthesis suggests a low-density Universe with the density parameter $\Omega \approx 0.05$ (this difficulty was already discussed by Zeldovich in the Tallinn 


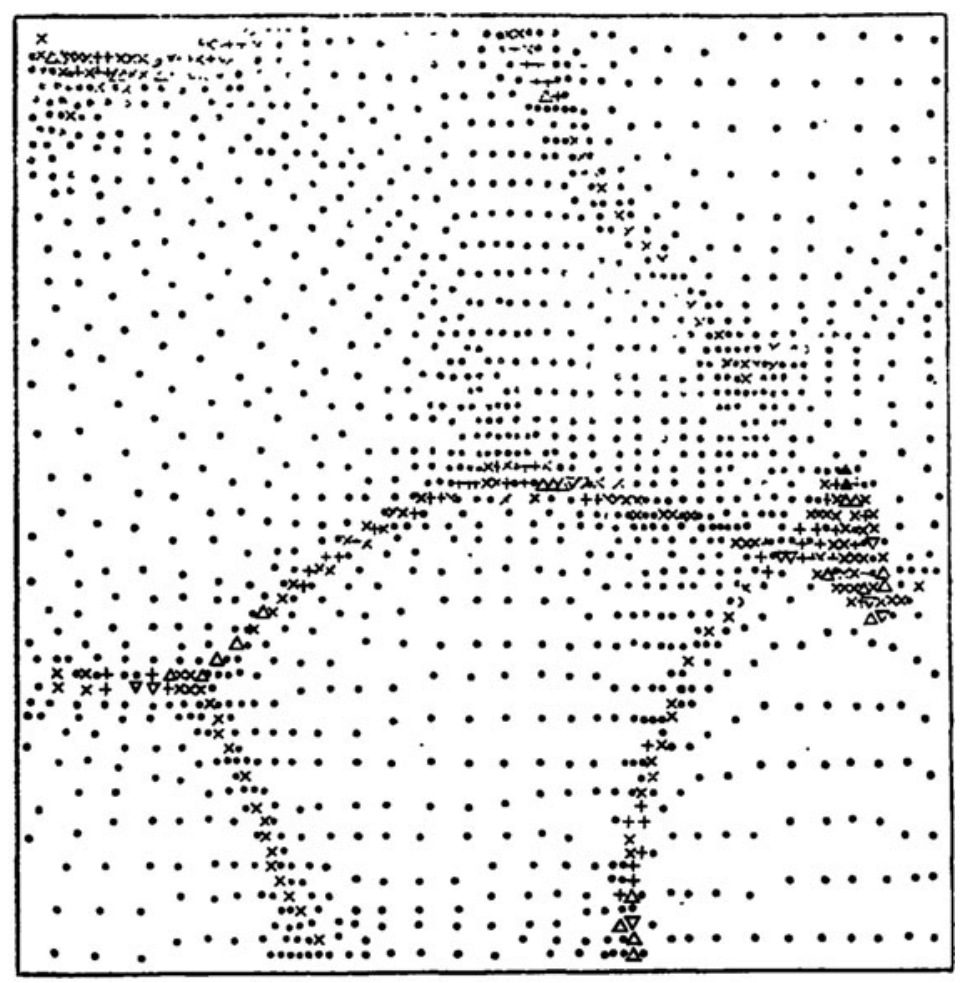

Figure 1. Distribution of particles in simulations according to Zeldovich pancake model, cited by Einasto et al.(1980).

conference); the smoothness of the Hubble flow also favours a low-density Universe. It was clear, that the existence of dark matter is in conflict with the classical cosmological paradigm. If it exists, then the density $\Omega \approx 0.2$ must be explained in some other way.

The nature of dark matter and its role in the evolution of the universe was a problem for almost ten years. To solve it data on the distribution of galaxies in space and other new data were needed.

\section{Structure of the Universe}

When Zeldovich asked the question on the formation of galaxies I had initially no idea how we could find an answer. But soon I remembered our previous experience in the study of galactic populations: their spatial distribution and kinematics evolve slowly. Systems of galaxies are much larger in size, thus their evolution must be even slower. Random velocities of galaxies are of the order of several hundred $\mathrm{km} / \mathrm{s}$ or less, thus during the whole lifetime of the Universe galaxies have moved from their place of origin only about $1 h^{-1} \mathrm{Mpc}$. If there exist some regularities in the large-scale distribution of galaxies, these regularities must reflect the conditions in the Universe during the formation of galaxies. Thus we had a leading idea to answer the second Zeldovich question: We have to study the distribution of galaxies on large scales.

We started to collect redshift data from all available sources. Since we needed data on large-scale distribution of galaxies, we collected redshifts not only for galaxies, but also for near cluster, both Abell and Zwicky clusters, as well as active galaxies (radio and Markarian galaxies). Our experience showed that clusters and active galaxies are good 

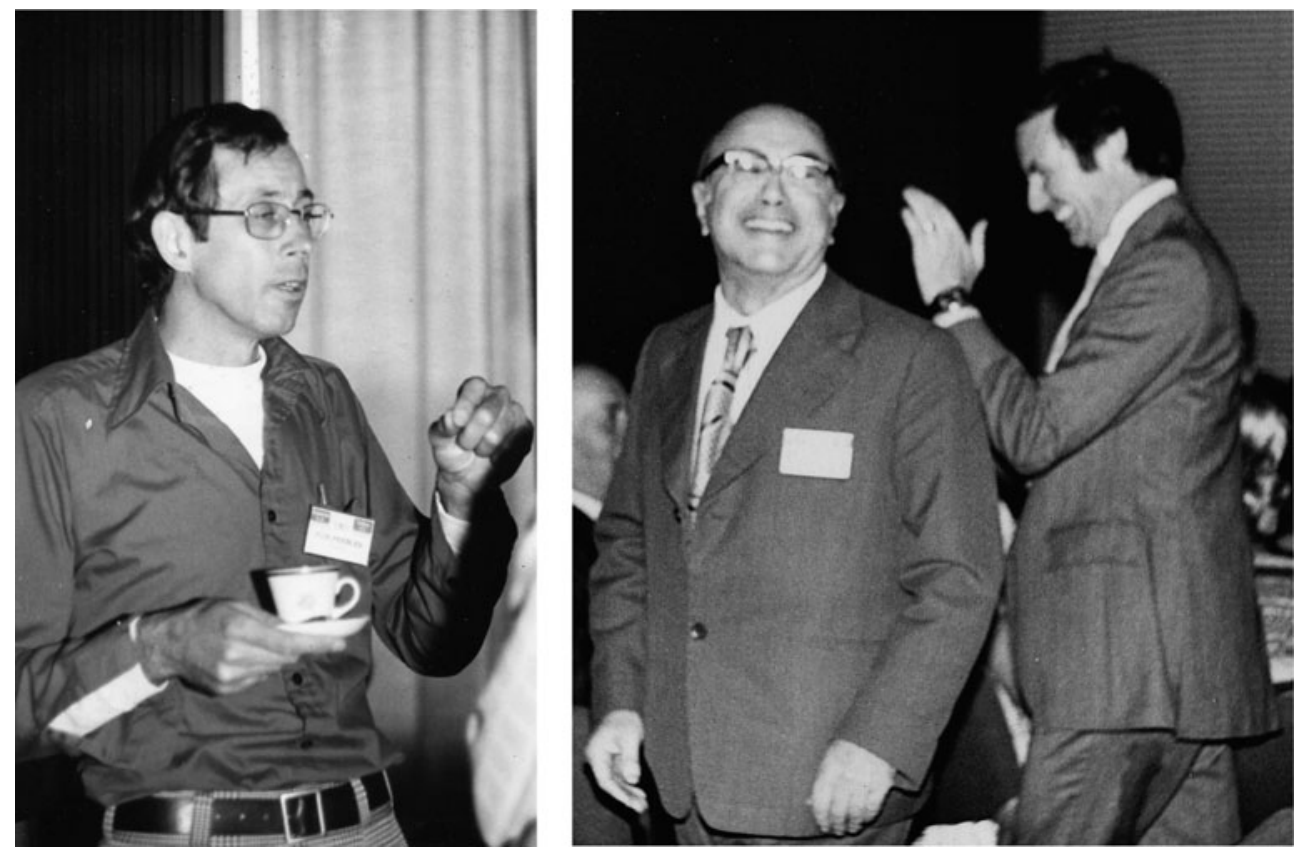

Figure 2. Jim Peebles (left), Yakov Zeldovich and Malcolm Longair (right) at the IAU Tallinn Symposium 1977.

tracers of the skeleton of the structure. Redshifts of galaxies and clusters were searched for the whole Northern Hemisphere.

In the middle of 1970's there were two basic structure formation scenarios, the Peebles \& Yu (1970) hierarchical clustering scenario and the Zeldovich (1970) pancake scenario. The hierarchical scenario represents well the apparent 2-dimensional distribution of galaxies, seen in Lick maps. Numerical experiments done in the Zeldovich team showed the formation of high-density knots joined by chains of particles to a connected network. Our challenge was to find out whether the real distribution of galaxies shows some similarity with one of the theoretical pictures.

To visualise the three-dimensional distribution we used wedge diagrams at various declination intervals and plots of clusters of galaxies in the Perseus supercluster region at different redshift intervals.

After the Tbilisi Meeting Zeldovich proposed to organise an international symposium devoted solely to cosmology. This suggestion was approved by IAU, and the symposium "Large Scale Structure of the Universe" was hold in Tallinn in September 1977. Two pictures of participants are shown in Figure 2.

The first speaker on the distribution of galaxies was Tully \& Fisher (1978), who showed a film of the Local Supercluster. The film showed that the supercluster consists of a number of galaxy chains which branch off from the supercluster's central cluster. No galaxies could be seen in the space between the chains. The presence of voids in the distribution of galaxies was reported also by Tifft \& Gregory (1978), and Tarenghi et al. (1978) in the Coma and Hercules superclusters, respectively.

In our presentation we showed wedge diagrams and cluster plots obtained in the Perseus supercluster region, see Figure 3. In the Figure left column shows wedge diagrams in three declination zones (Jõeveer \& Einasto (1978)). Filled circles are for rich clusters of galaxies, open circles - groups, dots - galaxies, crosses - Markarian galaxies. In right 

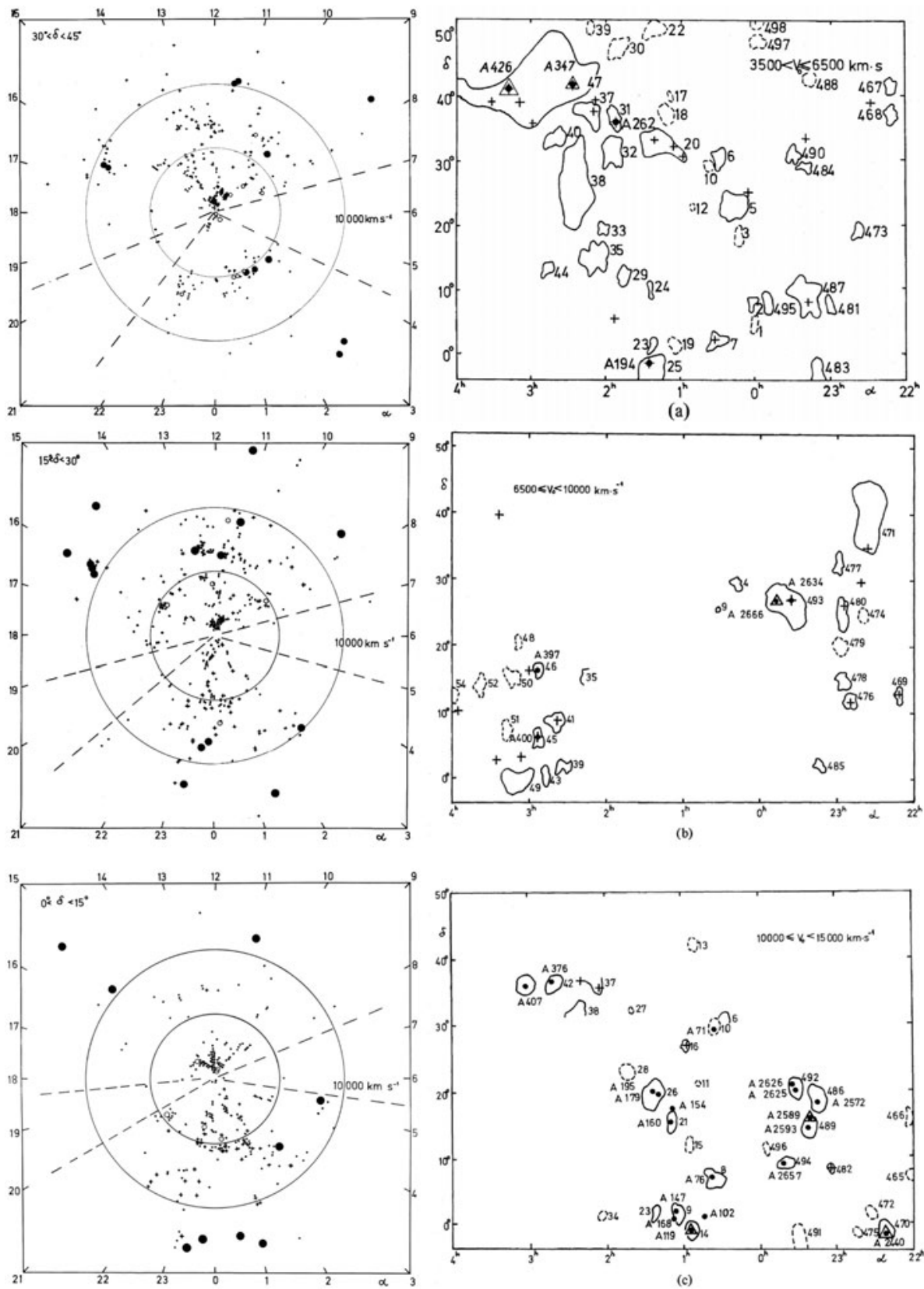

Figure 3. The distribution of galaxies and clusters, see text for explanations.

panels we plot Abell clusters and contours of Zwicky clusters in the Perseus area of sky at three distance intervals (Einasto et al. (1980)). When combined these plots show the large-scale three-dimensional distribution of galaxies and systems of galaxies.

These pictures showed a great richness of the distribution of galaxies - instead of random distribution of galaxies and clusters there exists a complicated hierarchical network, which we called "cellular structure". Not only filaments (chains) of galaxies and clusters were seen, but it was clear that galaxy chains form bridges between superclusters, thus 
there exists an almost continuous network of superclusters and filaments. Some chains are rich and consists of clusters and groups of galaxies, as the main ridge of the PerseusPisces supercluster. Filaments of galaxies across large voids are poor and consist only of galaxies and poor Zwicky clusters. In short, the three-dimensional data showed that the structure of the Universe is much richer than believed so far. Presently it is called the cosmic web.

Our picture had some similarity with the simulation made for the Zeldovich pancake scenario. However, the comparison was made only on the basis of visual impression by the comparison of the model and observed distributions of particles/galaxies. Zeldovich (1978) in his talk suggested to compare observations with models using quantitative methods. Thus we started with Zeldovich and his collaborators a search of quantitative methods to investigate properties of the distribution of galaxies.

Our main results were published by Zeldovich et al. (1982). Here we used the correlation function, the connectivity of systems of galaxies, the length of the largest system calculated for various linking lengths, and the multiplicity function of systems of galaxies. Comparison was made for a three-dimensional pancake model, hierarchical clustering model, Poisson model, and observations (a volume limited sample of galaxies including the Virgo supercluster). These tests showed that in most tests the pancake model is in good agreement with observations. In contrast, the hierarchical clustering model is in conflict with all tests, see Figure 4.

However, some differences between the pancake model and observations were evident. The most important difference is the lack of systems of intermediate richness in the pancake model, observed in real galaxy samples. As we understood soon, the reason for this disagreement was the assumption that dark matter consists of neutrinos.

\section{Astro-particle physics}

In early 1980's several important observational and theoretical analyses were made which reinforced the need for a paradigm shift. To understand the nature of dark matter of key value were searches of fluctuations of temperature fluctuations of CMB. From theoretical considerations it was clear that the temperature of CMB cannot be constant, and the expected amplitude of fluctuations was $\delta T / T \approx 10^{-3}$ (assuming the baryonic nature of the hot plasma before recombination). Fluctuations were searched with best radio telescopes available, none was found and the upper limits were much lower than the expected amplitude.

The most important theoretical development was the elaboration of the inflation model of the early universe by Starobinsky (1980), Starobinsky (1982), Guth (1981) and Linde (1982). The inflation model was based on various observational and theoretical considerations. One of the main conclusions of the model is the prediction that the total matter/energy density of the universe must be exactly equal to the critical cosmological density, $\Omega_{t o t}=1$.

The observed density of baryonic matter in the universe is about $\Omega_{b}=0.05$, supported by primordial nucleosynthesis considerations (mentioned already by Materne \& Tammann (1976) in the dark matter discussion in Tbilisi, 1975). Thus the only way to explain the low level of CMB temperature fluctuations, and conclusions from the inflation theory was to assume, that dark matter is non-baryonic. Non-baryonic matter is very weakly interacting with radiation, and density fluctuations in the non-baryonic matter can start to amplify already during the hot phase of the evolution of the universe.

These problems were discussed in April 1981 in a workshop in Tallinn, where both particle physicists and astronomers attended. A workshop of similar topic was held in 
September-October in Vatican. In both workshops one of the main conclusions was the non-baryonic nature of dark matter. These workshops mark the formation of a new area in research - astro-particle physics.

The first natural candidate for the dark matter was neutrino, the only known nonbaryonic particle. However, the problems with neutrinos as dark matter candidate were soon realised, as discussed, among others, by Zeldovich et al. (1982). Thus astronomers and physicists started to think what would be the alternatives. The main argument against neutrinos was their very high speed, close to the speed of light, which allowed to form only very massive cluster-sized systems. To allow the formation of smaller systems dark matter particles must have higher mass and lover speed. So various hypothetical particles were considered allowing the formation of systems of lower mass. Such particles were commonly called Cold Dark Matter, in contrast to neutrino-based Hot Dark Matter.

In 1983 Adrian Melott has made N-body simulations with density perturbation spectra which corresponded to the hot dark matter as well as to the cold dark matter scenario. He visited Moscow and Tallinn to discuss his results and to compare models with observations. The analysis was made jointly with Moscow and Tartu teams, and was published by Melott et al. (1983). Here we applied the same tests as used by Zeldovich et al. (1982). Our results showed that the CDM model is in excellent agreement with all quantitative tests. The paper ends with the conclusion, that the formation of the structure starts with the flow of particles to form the filamentary web as in the Zeldovich pancake model, but in the subsequent evolution systems grow as in the hierarchical clustering scenario by Peebles.

The advantages of the CDM model were discussed in detail by Blumenthal et al. (1984). Now, finally the presence of dark matter was accepted by leading theorists. A very detailed series of N-body simulations based on CDM and accepting a closed universe with critical density was made by the "Gang of Four" (Efstathiou et al. (1985), White et al. (1987)).

In 1980's the attention of our cosmology team in Tartu was devoted to quantitative study of the structure of the cosmic web using various tests. In these studies we used initially Melott CDM simulations to compare observation with models of structure formation. But we had the need to have our own simulations to have full control of the model. Enn Saar suggested to develop a model with critical cosmological density with dark matter fraction, $\Omega_{D M}=0.2$, as we have found from observations (Einasto et al. (1974)). The rest of the matter/energy density is in the cosmological $\Lambda$ term, $\Omega_{\Lambda}=1-\Omega_{D M}=0.8$. The simulation was made by our gradual student Mirt Gramann.

Our LCDM model was used to investigate various properties of the cosmic web. The first study was devoted to the topology of the cosmic web by Einasto et al. (1986). This study shows that the LCDM model fits observational data even better than the standard CDM model with critical density. The need to use the LCDM model was discussed in detail by Efstathiou et al. (1990.

Already in our first study of the cosmic web by Jõeveer et al. (1977) we had the question: Do galaxies form sheets between filaments as expected in the pancake scenario, or are they formed only in high-density regions as filaments at sheets crossing, and knots at filament crossings. The same question was asked also by Zeldovich et al. (1982), and studied on the basis of observational data by Einasto et al. (1980). The preliminary answer was - there exists no sheets of galaxies which isolate neighbouring low-density regions between superclusters.

The more detailed study by Einasto et al. (1986) showed, that the topology of the web depends on the threshold density level applied to separate low- and high-density regions in simulations. At very low threshold density sheets of particles isolate voids between rich 

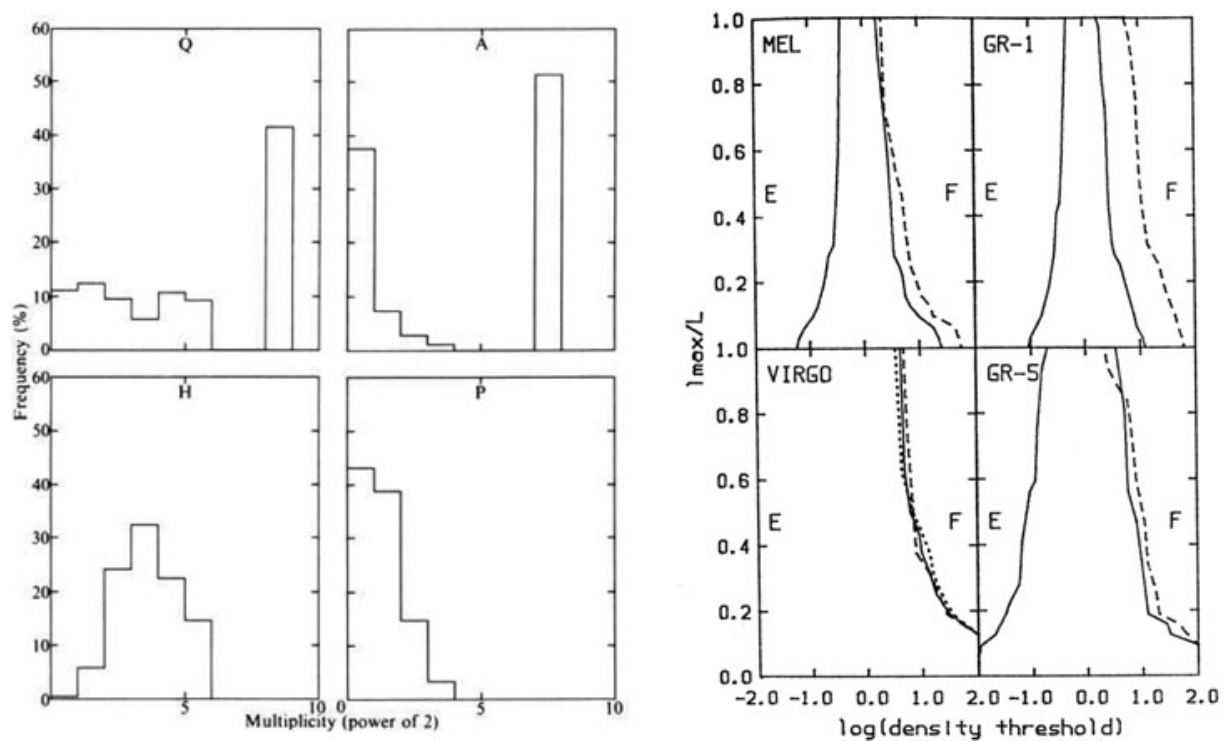

Figure 4. Left: the distribution of galaxies according to the multiplicity of the system. Multiplicity is expressed in powers of 2. Samples are designated: $\mathrm{O}$ - observed, A - adiabatic pancake model, H - hierarchical clustering model, P - Poisson model (Zeldovich et al. (1982)). Right: the length of the largest system (in units of the box size) versus the density threshold (in units of the mean density of the sample). $\mathrm{E}$ is for low density (empty) regions, F high density (filled) regions; MEL - Melott CDM simulation, GR-1 and GR-5 Gramann LCDM simulation at expansion factors 1 and 5.2 (present epoch), and VIRGO the observed sample around the Virgo supercluster. In models solid lines indicate unbiased samples with all test particles included, dashed lines biased samples, where particles in low-density regions have been removed (Einasto et al. (1986)).

regions. However, in low-density regions there exists no conditions to form galaxies. The density of the collapsing gas must exceed a threshold about 1.6 of the mean density to have during the Hubble time the possibility to collapse, as shown by Press \& Schechter (1974). If we exclude particles from low-density regions (biased galaxy formation) then voids (low-density regions in simulations) form just one large connected region, both in real and model samples - there are no sheets isolating voids, see Figure 4 . The length of the largest system depends on the threshold level in all samples. Similar results were obtained by Gott et al. (1986).

One aspect of the structure of the cosmic web is its fractal character, as suggested by Mandelbrot (1982), Mandelbrot (1986). During a visit to NORDITA with Enn Saar we investigated the fractal properties of the web in collaboration with Bernard Jones and Vicent Martinez. Ou results showed that both observational and model samples show multi-fractal properties (Jones et al. (1988)).

One difficulty with the original pancake scenario by Zeldovich was the shape of objects formed during the collapse. It was assumed that forming systems are flat pancake-like objects, whereas the dominant features of the cosmic web are filaments (Jõeveer \& Einasto (1978), Einasto et al. (1980)). This discrepancy was explained by Bond et al. (1996), who showed that, due to tidal forces, in most cases only essentially one-dimensional structures, i.e. filaments form. 


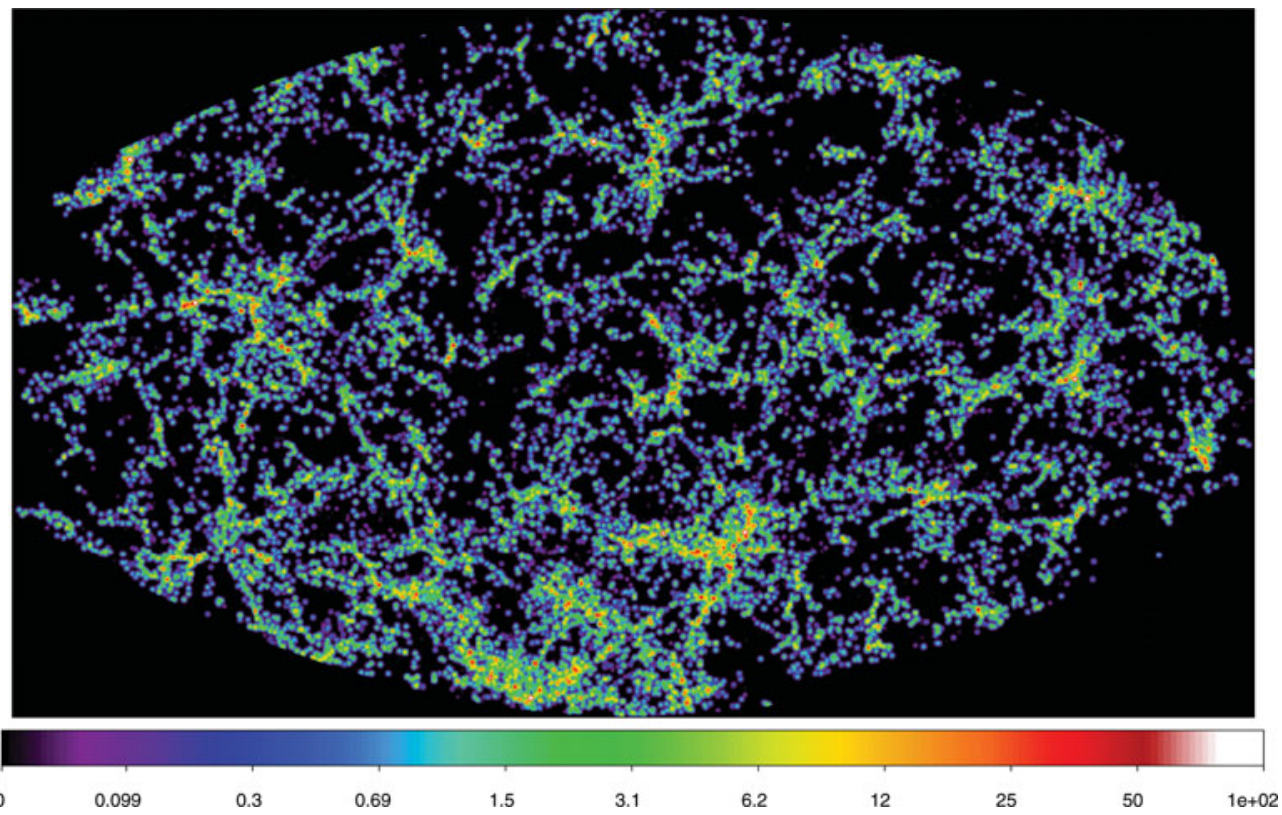

Figure 5. The luminosity density field of the SDSS in a spherical shell of $10 h^{-1} \mathrm{Mpc}$ thickness at a distance of $240 h^{-1}$ Mpc. The density scale is logarithmic, in units of the mean luminosity density for the whole DR7. The rich complex in the lower area of the picture is part of the Sloan Great Wall; it consists of three very rich superclusters (Suhhonenko et al. (2011)).

\section{Summary}

The contribution of the Zeldovich team to modern cosmology is impressive. Zeldovich was very actively collaborating with other groups, including our Tartu cosmology team. Thank to close collaboration with him and his collaborators we jointly succeeded to get interesting results on the nature of dark matter and the structure of the cosmic web, and the connection between these two phenomena.

What impressed me most in the new cosmological paradigm is its beauty - the Universe is much richer than thought before. The presence of dark matter shows that the Nature of the Universe is richer, in addition to known forms of matter it contains a new population, dark matter, the nature of which is not known even today. The structure of the Universe is also richer, instead of a random background of field galaxies we see now the cosmic web with all its small and large details.

I thank all my collaborators in Tartu and Moscow for very fruitful years of the search of properties of the universe.

\section{References}

Abell, G. O. 1958, ApJS, 3, 211

Abell, G. O., Corwin, Jr., H. G., \& Olowin, R. P. 1989, ApJS, 70, 1

Blumenthal, G. R., Faber, S. M., Primack, J. R., \& Rees, M. J. 1984, Nature, 311, 517

Bond, J. R., Kofman, L., \& Pogosyan, D. 1996, Nature, 380, 603

de Vaucouleurs, G. 1978, ApJ, 224, 710

Doroshkevich, A. G., Joeveer, M., \& Einasto, J. 1975, AZh, 52, 1113

Efstathiou, G., Davis, M., White, S. D. M., \& Frenk, C. S. 1985, ApJS, 57, 241 
Efstathiou, G., Sutherland, W. J., \& Maddox, S. J. 1990, Nature, 348, 705

Einasto, J. 1965, Trudy Astrophys. Inst. Alma-Ata (Tartu Astr. Obs. Teated 17), 5, 87

Einasto, J. 1974, in Stars and the Milky Way System, ed. L. N. Mavridis, 291

Einasto, J., Gramann, M., Einasto, M., et al. 1986, Tartu Astr. Obs. Preprint, A-9, 3

Einasto, J., Jõeveer, M., \& Saar, E. 1980, MNRAS, 193, 353

Einasto, J., Kaasik, A., \& Saar, E. 1974, Nature, 250, 309

Forman, W., Kellogg, E., Gursky, H., Tananbaum, H., \& Giacconi, R. 1972, ApJ, 178, 309

Friedmann, A. 1922, Zeitschrift fur Physik, 10, 377

Gott, III, J. R., Dickinson, M., \& Melott, A. L. 1986, ApJ, 306, 341

Guth, A. H. 1981, Physics Letters D, 23, 347

Hubble, E. 1929, Proceedings of the National Academy of Science, 15, 168

Hubble, E. P. 1925, ApJ, 62, 409

Jõeveer, M. \& Einasto, J. 1978, in IAU Symposium, Vol. 79, Large Scale Structures in the Universe, ed. M. S. Longair \& J. Einasto, 241-250

Jõeveer, M., Einasto, J., \& Tago, E. 1977, Tartu Astr. Obs. Preprint, 3

Jones, B. J. T., Martinez, V. J., Saar, E., \& Einasto, J. 1988, ApJL, 332, L1

Kuzmin, G. 1952, Tartu Astr. Obs. Publ., 32, 211

Kuzmin, G. 1956, AZh, 33, 27

Linde, A. D. 1982, Physics Letters B, 108, 389

Mandelbrot, B. B. 1982, The Fractal Geometry of Nature, ed. Mandelbrot, B. B.

Mandelbrot, B. B. 1986, Physics Today, 39, 11

Materne, J. \& Tammann, G. A. 1976, in Stars and Galaxies from Observational Points of View, ed. E. K. Kharadze, 455-462

Melott, A. L., Einasto, J., Saar, E., et al. 1983, Physical Review Letters, 51, 935

Oort, J. H. 1940, ApJ, 91, 273

Öpik, E. 1922, ApJ, 55, 406

Ostriker, J. P., Peebles, P. J. E., \& Yahil, A. 1974, ApJL, 193, L1

Peebles, P. J. E. 1971, Physical cosmology (Princeton Series in Physics, Princeton, N.J.: Princeton University Press, 1971)

Peebles, P. J. E. \& Yu, J. T. 1970, ApJ, 162, 815

Penzias, A. A. \& Wilson, R. W. 1965, ApJ, 142, 419

Press, W. H. \& Schechter, P. 1974, ApJ, 187, 425

Roberts, M. S. 1966, ApJ, 144, 639

Rubin, V. C. \& Ford, W. K. J. 1970, ApJ, 159, 379

Sandage, A. \& Tammann, G. A. 1975, ApJ, 196, 313

Shane, C. \& Wirtanen, C. 1967, Publ. Lick Obs., 22

Soneira, R. M. \& Peebles, P. J. E. 1978, AJ, 83, 845

Starobinsky, A. A. 1980, Physics Letters B, 91, 99

Starobinsky, A. A. 1982, Physics Letters B, 117, 175

Suhhonenko, I., Einasto, J., Liivamägi, L., et al. 2011, A\&SA, 531, A149

Sunyaev, R. A. \& Zeldovich, Y. B. 1969, Nature, 223, 721

Tarenghi, M., Tifft, W. G., Chincarini, G., Rood, H. J., \& Thompson, L. A. 1978, in IAU Symposium, Vol. 79, Large Scale Structures in the Universe, ed. M. S. Longair \& J. Einasto, 263

Tifft, W. G. \& Gregory, S. A. 1978, in IAU Symposium, Vol. 79, Large Scale Structures in the Universe, ed. M. S. Longair \& J. Einasto, 267

Tinsley, B. M. 1968, ApJ, 151, 547

Tully, R. B. \& Fisher, J. R. 1978, in IAU Symposium, Vol. 79, Large Scale Structures in the Universe, ed. M. S. Longair \& J. Einasto, 214

van den Bergh, S. 1972, A\& A, 20, 469

White, S. D. M., Frenk, C. S., Davis, M., \& Efstathiou, G. 1987, ApJ, 313, 505

Zeldovich, Y. B. 1970, A\&SA, 5, 84 
Zeldovich, Y. B. 1978, in IAU Symposium, Vol. 79, Large Scale Structures in the Universe, ed. M. S. Longair \& J. Einasto, 409-420

Zeldovich, Y. B., Einasto, J., \& Shandarin, S. F. 1982, Nature, 300, 407

Zwicky, F. 1933, Helvetica Physica Acta, 6, 110

Zwicky, F., Herzog, E., \& Wild, P. 1968, Catalogue of galaxies and of clusters of galaxies (Pasadena: California Institute of Technology (CIT), 1961-1968) 